\title{
Solenoid Actuator Design for Improvement of Response
}

\section{Speed}

\author{
Baek Ju Sung \\ Reliability Assessment Center, Korea Institute of Machinery \& Materials, Daejeon 34103, Korea
}

Received: March 17, 2016 / Accepted: March 22, 2016 / Published: May 31, 2016.

\begin{abstract}
The main design factors which effect on operating speed of solenoid actuator for valve operation are mass of plunger, electromagnetic motive force, inductance and return spring, and these factors are not independent but related with each other in view point of design and electromagnetic theory. It is impossible to increase the operating speed by only change the value of any one design factor. The change of any one value results in change of any value related it in various design factors. Permanent magnets are as assistant materials which make higher flux density in air gap. Electromagnetic motive force in controlled only by current. This paper presents a speed increasing design method of solenoid actuator using a solenoid, by some governing equations which are composed of electromagnetic theory and empirical knowledge, and proved the propriety by experiments.
\end{abstract}

Key words: Solenoid actuator, high speed, servo valve, permanent magnet, non-magnetic ring, plunger, yoke, performance test, attraction force, frequency response.

\section{Introduction}

Solenoid actuator is a very economical motion converter due to its simple structure. For optimal design, theoretical and empirical knowledge are simultaneously needed. Theoretical knowledge governs the operational characteristics of the solenoid actuator, and empirical knowledge compensates for the theoretical limitation obtained from the designer's design and manufacturing experiences for various kinds of solenoid actuators [1].

In particular, empirical knowledge is more essential than theoretical knowledge for determination of the plunger shape and value of the space factor, because the varieties of plunger shape are very versatile and the space factor has a subjective property. So, they cannot be determined solely by calculation or simulation. When designer's accumulated experiences and expertise are added to theses, the most proper shape and value of them can then be obtained.

Permanent magnet is also an important component

Corresponding author: Baek Ju Sung, principal researcher, research fields: electrical actuator, reliability research and machinery components. effecting on operation of solenoid actuator. It needs the determination of optimal operating point and selection of magnetic material. But, in this study, we deal with the permanent magnet as an only flux density body having a constant value for simplification of design. So, the overall needed attraction force is controlled by current adjustment.

In this study, we derived the governing equations and composed a design program, and proved the propriety of them through FEM (finite elements method) analysis and various experiments by use of a prototype solenoid actuator.

\section{Governing Equations}

\subsection{The Structure of High Speed Solenoid Actuator}

Fig. 1 represents the simplified structure of high speed solenoid actuator, where the permanent magnets are excluded. Permanent magnet independently compensates the electromagnetic force of solenoid coil, and it contributes the reduction of consumption power and increasing of operational speed in comparison with the case of only used solenoid coil. 


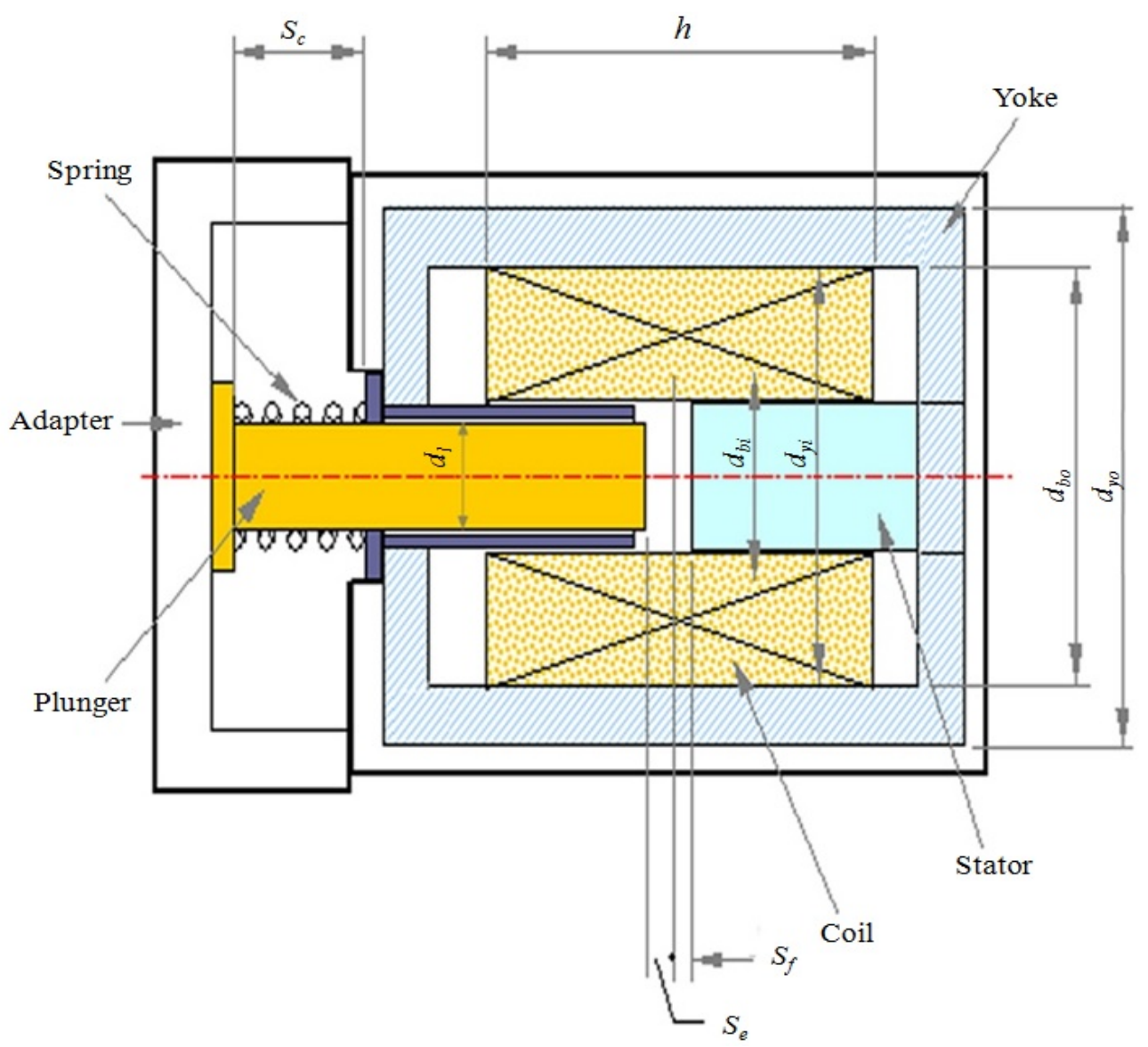

Fig. 1 Simple structure of solenoid actuator.

\subsection{Magnetic Flux Density and Magnetic Motive} Force in Air Gap

The attraction force $F$ is shown in Eq. (1) in the magnetic circuit of Fig. 2 [1-4].

$$
F=\frac{B^{2} \cdot S}{2 \mu_{0}}(N)
$$

where, $B$ is magnetic flux density, $S$ is cross sectional area of plunger, as it were, it is $\pi\left(\frac{d_{l}}{2}\right)^{2}$ when $d_{l}$ is radius of plunger, and $\mu_{0}$ is permeability in the air. Therefore from Eq. (1), the magnetic flux density $B$ needed in air gap is expressed as Eq. (2):

$$
B=\sqrt{\frac{F \cdot 2 \mu_{0}}{S}}
$$

And theoretical magneto motive force $U_{m}$ are shown in Eq. (3), and $d$ is maximum distance between plunger and stationary.

$$
U_{m}=\frac{B \cdot d}{\mu_{0}}
$$

Eq. (4) is obtained from Eqs. (1) and (3), and also, the design coefficient $K_{f}$ can be expressed as Eq. (5):

$$
F=\frac{K_{f}}{d^{2}}
$$

$$
K_{f}=\frac{\mu_{0} \cdot S \cdot U_{m}^{2}}{2}
$$

When the length of fixed air gap is $S_{f}$ in Fig. 2, the maximum distance $d$ between plunger and stationary is given to Eq. (6), that is represented by the sum of fixed air gap $S_{f}$ and plunger stroke $S_{e}$. So, the maximum attraction force $F_{\max }$ and the minimum attraction force $F_{\min }$ become Eqs. (7) and (8), respectively. 


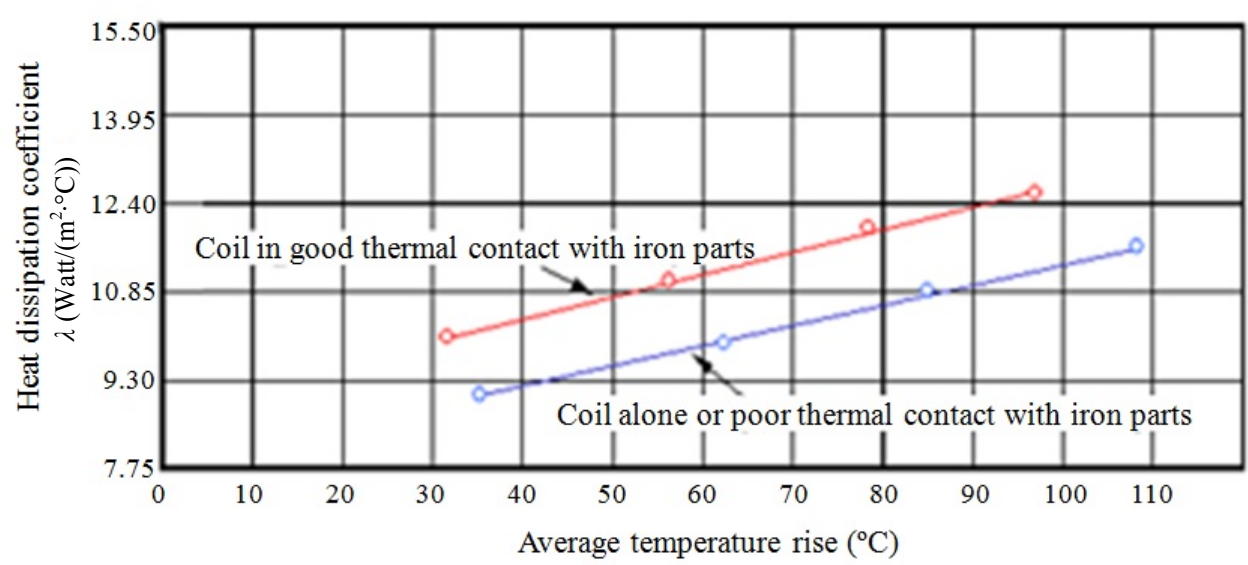

Fig. 2 Heat dissipation coefficient according to temperature rising.

$$
\begin{gathered}
d=S_{f}+S_{e} \\
F_{\text {max }}=\frac{K_{f}}{S_{f}^{2}} \\
F_{\text {min }}=\frac{K_{f}}{d^{2}}
\end{gathered}
$$

And therefore, form Eqs. (7) and (8), the relation between maximum attraction force and minimum attraction force become Eq. (9).

$$
F_{\max }=\left(\frac{d}{S_{f}}\right)^{2} \cdot F_{\min }
$$

Magnetic flux density of Eq. (3) is equal to Eq. (10) by substitution of Eqs. (1) and (8) to Eq. (9).

$$
B=2 \cdot \frac{\sqrt{2 \cdot \mu_{0} \cdot F_{\min }}}{d_{l} \cdot \sqrt{\pi}}
$$

And, from Eq. (3), the actual magneto motive force $U$, needed by the solenoid actuator, is obtained by Eq. (11).

$$
U=\frac{C_{m} \cdot B \cdot d}{\mu_{0}}
$$

where, $C_{m}$ is empirical compensation coefficient for magnetic motive force $[1,5]$. It is needed for compensating the loss portion of magnetic force in the actual magnetic circuit.

\subsection{Permanent Magnet and Flux Density in Air Gap}

By Refs. $[6,7]$, the total magnetic flux density in the air gap generated as Eq. (12) [6, 7].

$$
B_{g}=\frac{B_{r} \cdot h_{M}}{\frac{A_{g}}{A_{m}} h_{M}+\mu_{M} S_{f}}
$$

where, $A_{g}$ is cross sectional area of air gap, $A_{m}$ is pole area of permanent magnet, $B_{r}$ is residual magnetic flux density, $h_{M}$ is length of permanent magnet in magnet sticking direction.

And the permeability of permanent magnet $\mu_{M}$ is like Eq. (13).

$$
\mu_{M}=\frac{B_{r}}{H_{c} \mu_{0}}
$$

From Eq. (12), we can know that, the magnetic flux density of air gap approaches to the residual magnetic flux density when the length of permanent magnet is long and the length of air gap is completely short.

For decision of operating point of permanent magnet, we must consider the maximum energy area of permanent magnet and the reduced magnetic flux due to reaction of magnetic field by solenoid coil. But, in this paper, the change of characteristic of permanent magnet may not be occurred, because the operating point of permanent magnet resulted from completely short length of air gap and path of magnetic flux.

\subsection{Estimation of Yoke Thickness}

Referring Fig. 1, inner diameter $d_{y i}$ and outer diameter $d_{y o}$ of yoke are as Eqs. (14) and (15). 


$$
\begin{gathered}
d_{y i}=d_{b o}+C_{g} \\
d_{y o}=\sqrt{d_{y i}^{2}+C_{p} \cdot d_{l}^{2}}
\end{gathered}
$$

$$
\text { Yoke thickness }=\left(d_{y o}-d_{y i}\right) / 2
$$

The empirical constant $C_{g}$ in Eq. (14) is the length margin for smooth heat dissipation of the coil, and the empirical constant $C_{p}$ in Eq. (15) is the length margin for smooth passing of magnetic flux [8-10]. The proper values of the experience coefficients can be decided by designer's judgment depend on his experience and electromagnetic knowledge.

\subsection{Temperature Rising and Bobbin Length}

Heat dissipation coefficient $\lambda$ is the amount of heat energy radiated form the coil surface. It can be founded in Fig. 2 [1].

$R$ and $I$ passing through it produce the $T_{f}$ in Eq. (17). By substituting Eqs. (18) and (19) into Eq. (17), we can make the constructive equation of final temperature rising as Eq. (20). Eq. (17) is usually used temperature rising equation in the coil [1].

$$
\begin{gathered}
T_{f}=\frac{W}{2 \cdot \lambda \cdot S}=\frac{I^{2} \cdot R}{2 \cdot \lambda \cdot S} \\
R=\rho \frac{\left(l_{m} \cdot N^{2}\right)}{h \cdot w \cdot X_{i}} \\
X_{i}=\frac{\pi}{4}\left(\frac{d_{s}}{d_{0}}\right)^{2} \\
T_{f}=\frac{q \cdot \rho}{d \cdot \lambda \cdot X_{i} \cdot w} \cdot\left(\frac{N \cdot W}{h \cdot V}\right)^{2}
\end{gathered}
$$

where, $R$ is coil resistance, $I$ is current, $T_{f}$ is rising temperature, $S$ is area of heat dissipation $\left(=l_{m} \cdot h\right)$, $W$ is consumption power, $\rho$ is relative resistance, $l_{m}$ is coil mean length per single turn, $N$ is total turn numbers, $h$ is coil height, $w$ is coil layer thickness, $q$ is duty ratio, $d_{s}$ is diameter of bare wire, $d_{0}$ is diameter of insulated coil and $V$ is supply voltage.

Bobbin length (coil height) used in Eq. (18) is calculated by Eq. (21)

$$
h=\sqrt[3]{\frac{\left(q \cdot \beta \cdot \rho \cdot U^{2}\right)}{2 \cdot \lambda \cdot X_{i} \cdot T_{f}}}
$$

where, $\beta$ is the ratio of bobbin height $h$ and coil layer thickness $w$. That is, $\beta$ is equal to $\frac{h}{w}$, which is shown in the detailed drawing of the bobbin and yoke in Fig. 1 [8].

\subsection{Number of Turns and Consumption Power of Coil}

Mean length of coil $l_{m}$ turn is represented as Eq. (22):

$$
l_{m}=\frac{\pi\left(d_{b o}+d_{b i}\right)}{2}
$$

And the relation between equivalent resistance $R_{t}$ of solenoid circuit using copper wire, supply voltage $V$, current $I$ and relative resistance $\rho$ and be expressed by Eq. (23).

$$
R_{t}=\frac{V}{I}=4 \rho\left[\frac{\left(l_{m} \cdot N\right)}{\pi \cdot d_{s}^{2}}\right]
$$

Diameter of bare wire, $d_{s}$ is induced to Eq. (24) from Eq. (23).

$$
d_{s}=\sqrt{\left[\frac{2 \cdot \rho \cdot\left(d_{b o}+d_{b i}\right) \cdot U}{V}\right]}
$$

If it is assumed that, insulated wire diameter is $d_{0}$ and the winding loss of a winding layer is 1 turn, the total turn number to be winded $n_{c}$ in shaft direction given in Eq. (25). And, the total layer number $m_{c}$ of coil in the radial direction is given by Eq. (26).

$$
\begin{gathered}
n_{c}=\left(\frac{h}{d_{0}}\right)-1 \\
m_{c}=\frac{w}{d_{0}}
\end{gathered}
$$

Therefore the total turn number $N$ to be winded on the bobbin can be given by Eq. (27).

$$
N=n_{c} \cdot m_{c}
$$

By combining Eqs. (23) and (24), the equivalent 


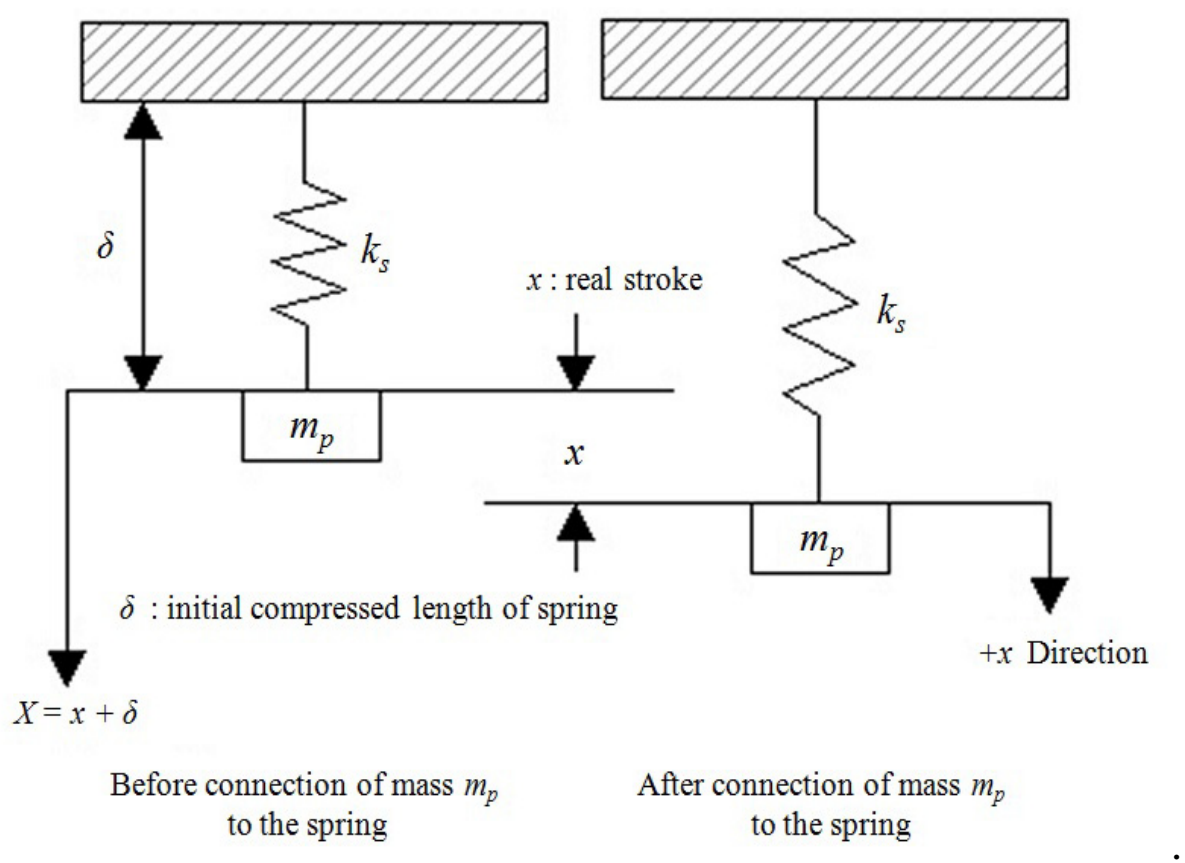

Fig. 3 Mechanical model of solenoid actuator.

resistance $R_{t}$, which represents the total resistance of coil, is fully obtained by Eq. (28).

$$
R_{t}=\frac{2 \cdot \rho \cdot\left(d_{b o}+d_{b i}\right) \cdot N}{\pi d_{s}^{2}}
$$

According to determination of $R_{t}$, the equations of coil current $I$ and consumption power $W$ are determined by Eqs. (29) and (30), respectively:

$$
\begin{gathered}
I=\frac{V}{R_{t}} \\
W=V \cdot I
\end{gathered}
$$

\subsection{Operating Frequency}

The solenoid actuator can be expressed as return spring - mass system like Fig. 3. After applying power, plunger displacement is equivalent to the displacement of mass $m_{p}$ [4].

The state equation of mass $m_{p}$ to the $\mathrm{x}$-direction is Eq. (31). By substitution of $X=x+\delta$ to Eq. (31), we can achieve Eq. (32):

$$
\begin{gathered}
m_{p} \ddot{X}+k_{s} X=m_{p} g \\
m_{p} \ddot{x}+k_{s} x=0
\end{gathered}
$$

Therefore, mathematical model about the system of
Fig. 4 becomes to Eq. (33):

$$
\ddot{x}+\omega^{2} x=0
$$

Here, the operating speed $\omega$ and operating frequency $f_{p}$ of the actuator can be expressed by Eqs. (34) and (35), respectively [4].

$$
\begin{gathered}
\omega=\sqrt{\frac{k_{s}}{m_{p}}} \\
f_{p}=\frac{\omega}{2 \pi}
\end{gathered}
$$

\section{Design Program}

The target specifications of prototype actuator for the hydraulic valve are as shown in Table 1.

Fig. 4 shows the flow chart of the developed design program which is programmed by use of the governing equations and empirical coefficients in Chapter 2.

Designer's judgment means designer's experience which is needed to judge the fact whether the final design parameters are proper for manufacturing of target actuator or not.

The input parameters and their values needed for design of the prototype are introduced in Table 2. 
Table 1 Target specifications.

\begin{tabular}{ll}
\hline Items & Target performance \\
\hline Supply voltage & $24 \mathrm{~V}$ \\
Consumption power & $55 \mathrm{~W}$ \\
Operating frequency & $100 \mathrm{~Hz}$ \\
Attraction force & $160 \mathrm{~N}$ \\
\hline
\end{tabular}

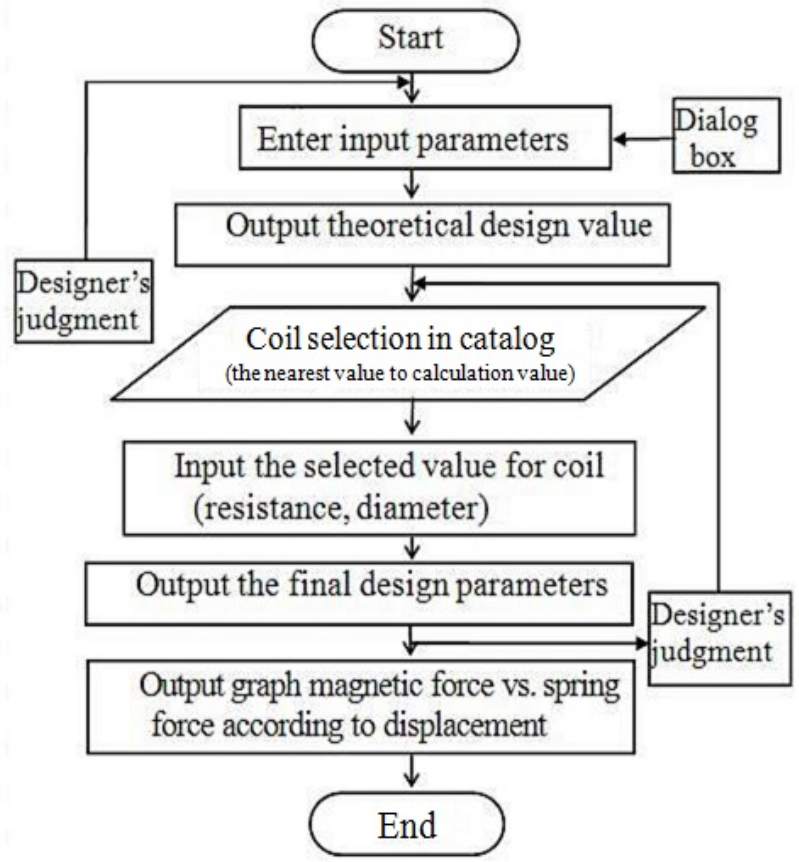

Fig. 4 Flow chart of design program.

\section{Table 2 Input parameters.}

\begin{tabular}{ll}
\hline Items & Input value \\
\hline$F_{\min }(\mathrm{N})$ & 160 \\
$d_{l}(\mathrm{~mm})$ & 4 \\
$t_{b}(\mathrm{~mm})$ & 1 \\
$n_{c}$ & 59 \\
$m_{c}$ & 8 \\
$C_{g}$ & 0.004 \\
$C_{p}$ & 1.25 \\
$V \quad(\mathrm{~V})$ & 24 \\
$m_{p}(\mathrm{~g})$ & 9.4 \\
$k_{s}(\mathrm{kgf} / \mathrm{mm})$ & 4.5 \\
\hline
\end{tabular}

By input of the values in Table 2 to the design program, we can obtain the results of Table 3 as a numerical output.
Table 3 Result of design.

\begin{tabular}{ll}
\hline Items & Output value \\
\hline Attraction force & $160(\mathrm{~N})$ \\
Magnetic flux density & $2.334(\mathrm{~T})$ \\
Bobbin inner diameter & $38(\mathrm{~mm})$ \\
Bobbin outer diameter & $50(\mathrm{~mm})$ \\
Turn number of coil & 502.775 \\
Yoke inner diameter & $50.004(\mathrm{~mm})$ \\
Yoke outer diameter & $54.17(\mathrm{~mm})$ \\
Equivalent resistance & 10.95 \\
Coil current & $2.19178082(\mathrm{~A})$ \\
Magnetic motive force $\left(\right.$ at $\left.20^{\circ} \mathrm{C}\right)$ & $1,101.9726(\mathrm{~A} . \mathrm{T})$ \\
Consumption power $\left(\right.$ at $\left.20^{\circ} \mathrm{C}\right)$ & $52.6027397(\mathrm{~W})$ \\
Operating frequency & $110(\mathrm{~Hz})$ \\
\hline
\end{tabular}

\section{FEM Analysis}

The FEM analysis is performed by use of on the design results of earlier chapter. Fig. 5 shows the modeling of solenoid actuator for three-dimension analysis. Fig. 6 represents the magnetic flux density in case of existing the permanent magnet in magnetic path. In this study, the used permanent magnet is $1 \mathrm{~T}$ class $\mathrm{NdFeB}$ for generation of appropriate thrust force in air gap, which has higher residual flux density by comparison to its lower price.

By referring to Fig. 6, the magnetic flux density of left and right side is same when the current is 0 . So, the thrust force of attraction force is not generated.

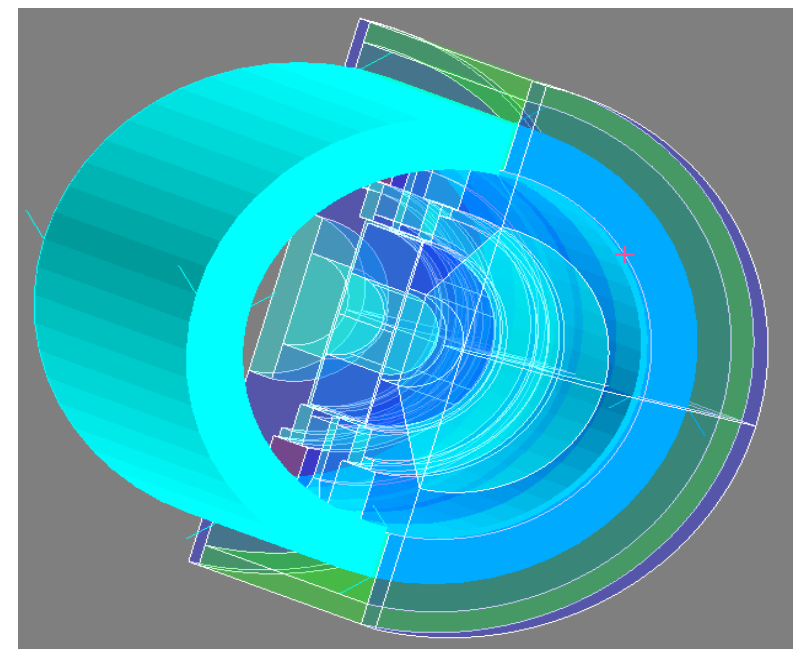

Fig. 5 Modeling of solenoid actuator. 


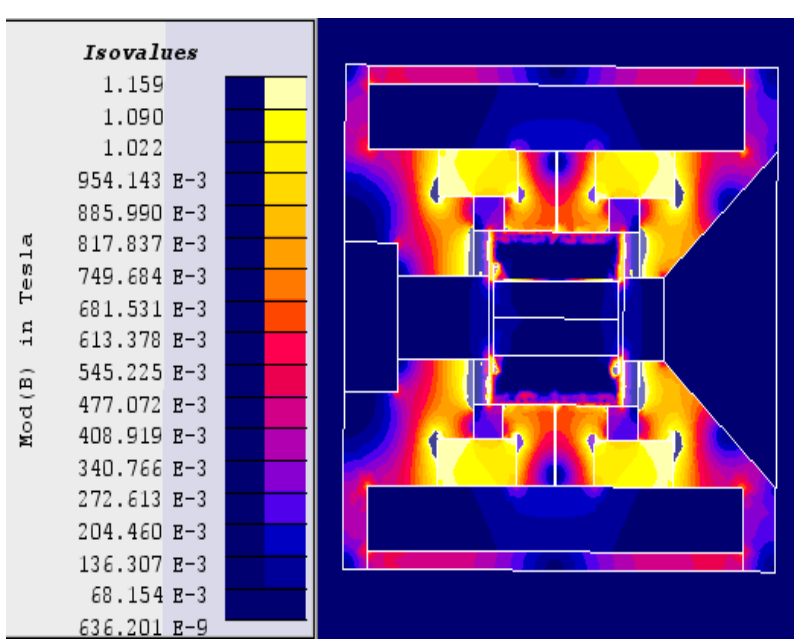

Fig. 6 Distribution of magnetic flux density considering only permanent magnet.

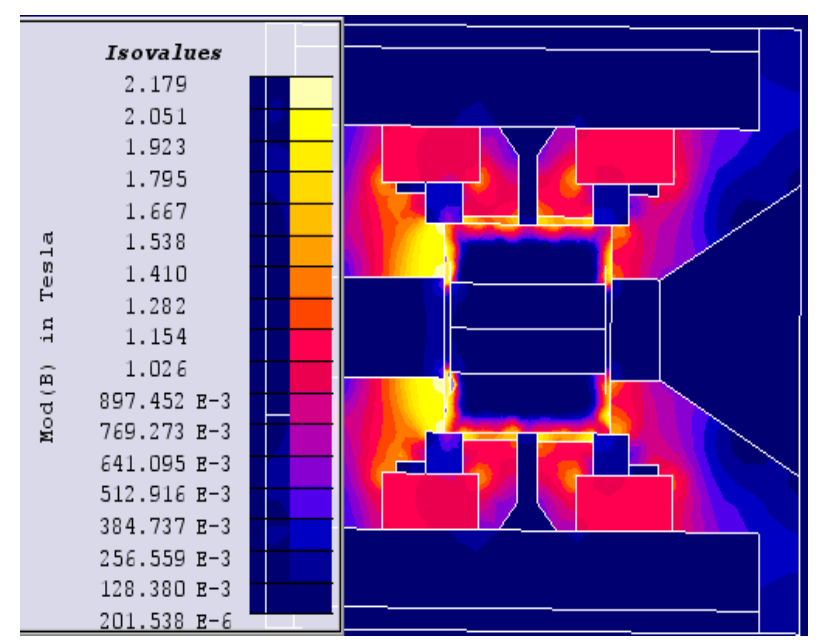

Fig. 7 Distribution of magnetic flux at $2.2 \mathrm{~A}$.

Fig. 7 shows the distribution of magnetic flux in air gap at rated current 2.2 A. At this time, the magnetic fields generated by solenoid and permanent magnet are added in air gap, so, the intensity of magnetic field in left side air gap becomes stronger and right side becomes weaker. As a result, the plunger should move to left direction by the attraction force of left side.

If the direction of current is conversely changed, the plunger may move to right direction on the same principle.

In Fig. 7, we can also know that, the combined magnetic flux density of solenoid and permanent magnet increases up to $2.2 \mathrm{~T}$ in around of left side air gap.
Fig. 8 is cut plane to observe the magnetic flux density in left side air gap of plunger in Fig. 7, Fig. 9 shows the magnetic flux density in this case.

In Fig. 9, the each regional locations generating the magnetic flux density are as follows: 7-13 is the region of permanent magnet installed in left top of Fig. 6, 13-24 is the region of air gap in plunger top located under 7-13 section, 28-36 is the region on air gap of plunger bottom, and 38-44 is the region of permanent magnet installed in left bottom of Fig. 6 .

By these analyses, we can know that, magnetic flux density of air gap, which make move the plunger, is up to $2.2 \mathrm{~T}$ when the rated current is supplied.

\section{Manufacturing of Prototype}

The prototype is manufactured by based on the design results and FEM analysis in previous chapters. Solenoid in made by plastic bobbin and copper coil, plunger is made by processing core steel, and yoke tube is installed for offering the smooth magnetic flux path between these. And, the permanent magnets are installed in front and rear of plunger for more increasing the operating speed.

For preventing the sticking of plunger onto the core tube, non-magnetic bushings of which thickness is 0.3-0.5 $\mathrm{t}$ is inserted in front and rear of the core tube. The permanent magnets must put together to be confronted the same magnetic poles, at here, we put together the $S$ poles are to be confronted by base of

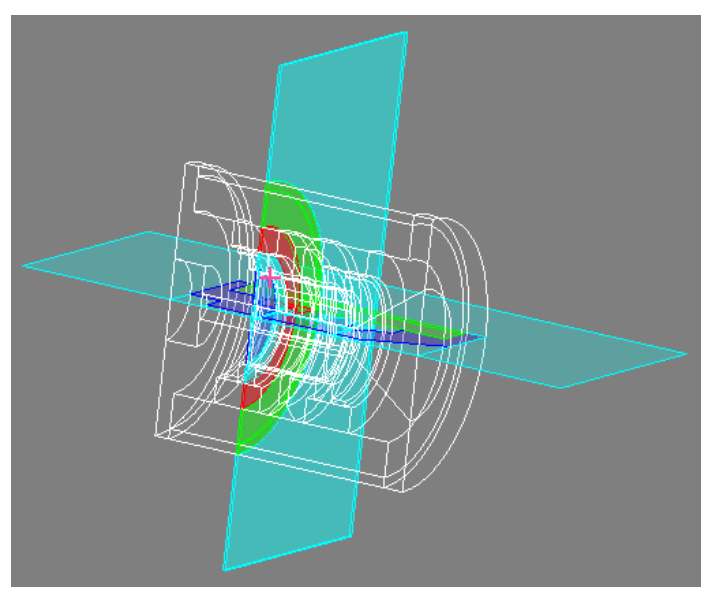

Fig. 8 Cut plane to observe the distribution of magnetic flux density. 


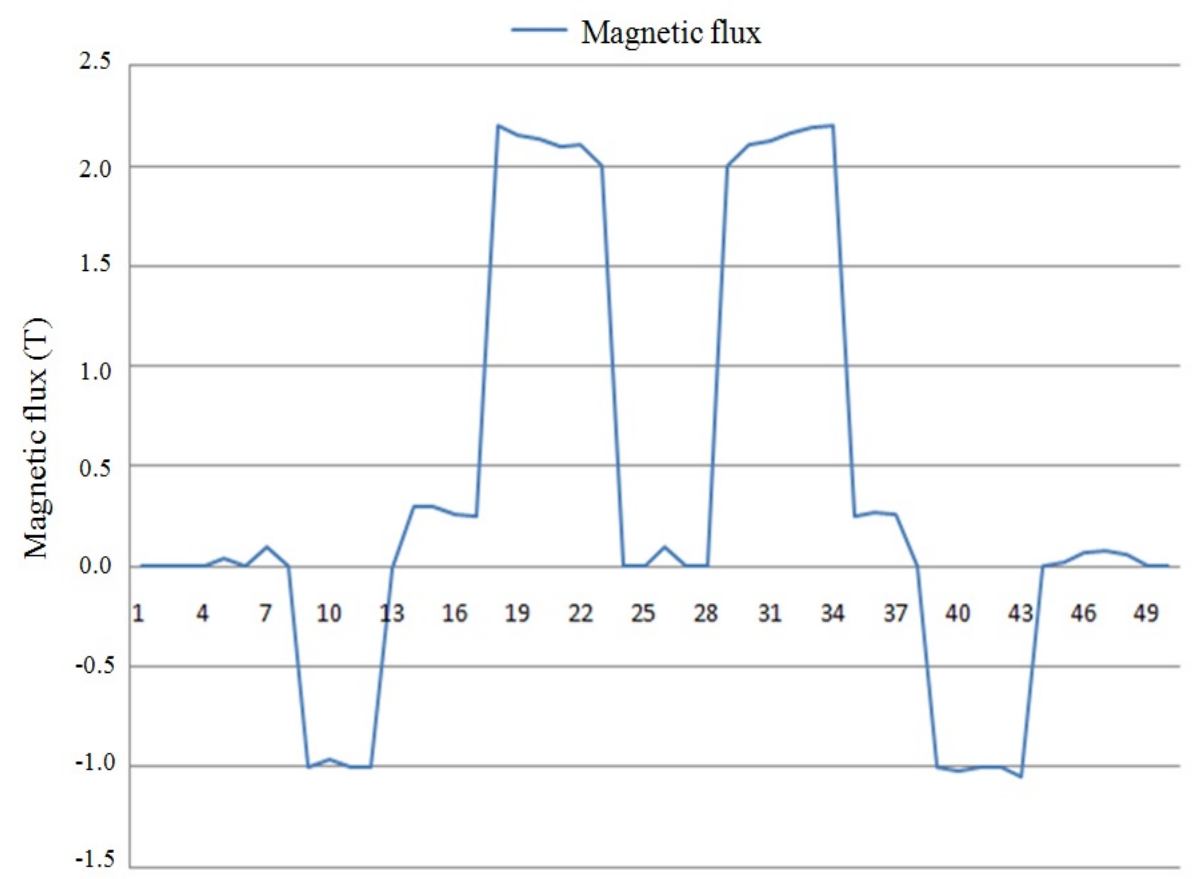

Plane number

Fig. 9 Distribution of magnetic flux density in left side air gap of plunger using Fig. 8.

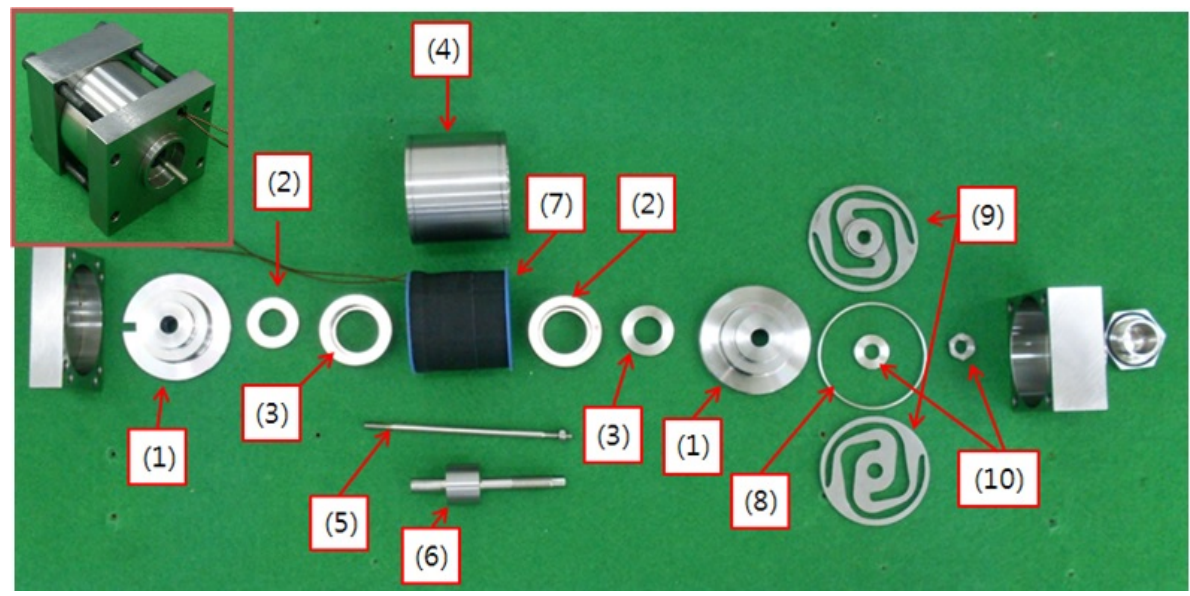

\begin{tabular}{|l|l|l|l|}
\hline $1:$ & Core tube & $6:$ & Plunger \\
\hline $2:$ & Bushing & $7:$ & Bobbin and coil \\
\hline $3:$ & Permanent magnet & $8:$ & Ring \\
\hline $4:$ & Yoke tube & $9:$ & Centering spring \\
\hline $5:$ & $\begin{array}{l}\text { Core and supporter for LVDT (linear } \\
\text { variable differential transformer) }\end{array}$ & $10:$ & Washer \\
\hline
\end{tabular}

Fig. 10 Main components of prototype \& assembly.

plunger. And, two pieces of centering springs are installed for security of neutral position of plunger in both side permanent magnets.

In addition, solenoid bearing is inserted in front of core tube. This plays a role to minimize the friction force and make the plunger locate in spatial center of inner core tube. Push rod is unified with plunger by laser welding after pressing, the rod bar transfers the attraction force of plunger to spool.

The components of manufacturing prototype and the 
assembly show in Fig. 10.

We used a ready-made controller for test of prototype in this paper. The rated input voltage is $24 \mathrm{Vdc}$, maximum consumption power is $60 \mathrm{~W}$, control signal voltage is $\pm 10 \mathrm{Vdc}$, and the variable output current is maximum $8 \mathrm{~A}$.

\subsection{Test of Step Response}

This test is to measure the time difference between supplying time of input step signal and reaction time of plunger. Here, the $100 \%$ control signal $(10 \mathrm{~V})$ to controller is used as input step signal and reaction of plunger is detected by output signal of LVDT.
Referring to Fig. 11, the control signal $10 \mathrm{~V}$ is applied to controller at point of time $57 \mathrm{~ms}$, and the reaction signal $2.2 \mathrm{~V}$ of LVDT is detected at point of time $62 \mathrm{~ms}$. So, the step response time is $5 \mathrm{~ms}$.

\subsection{Test of Frequency Response}

This test is similar to the test of step response. The input is control signal of controller and output is reaction signal of LVDT. This test performed at $25 \%$ magnitude of input signal with $0.01-200 \mathrm{~Hz}$ carrier frequency region. Fig. 12 is the test results for $25 \%$ control signal of controller. It shows that, the $-3 \mathrm{~dB}$ frequency is about $191 \mathrm{~Hz}$.

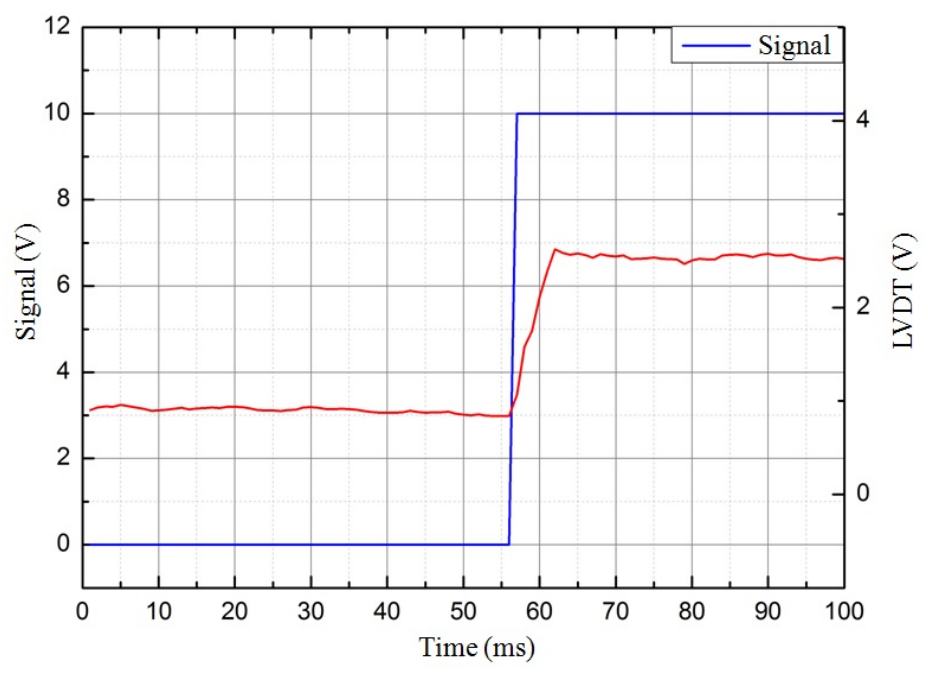

Fig. 11 Test result of step response.

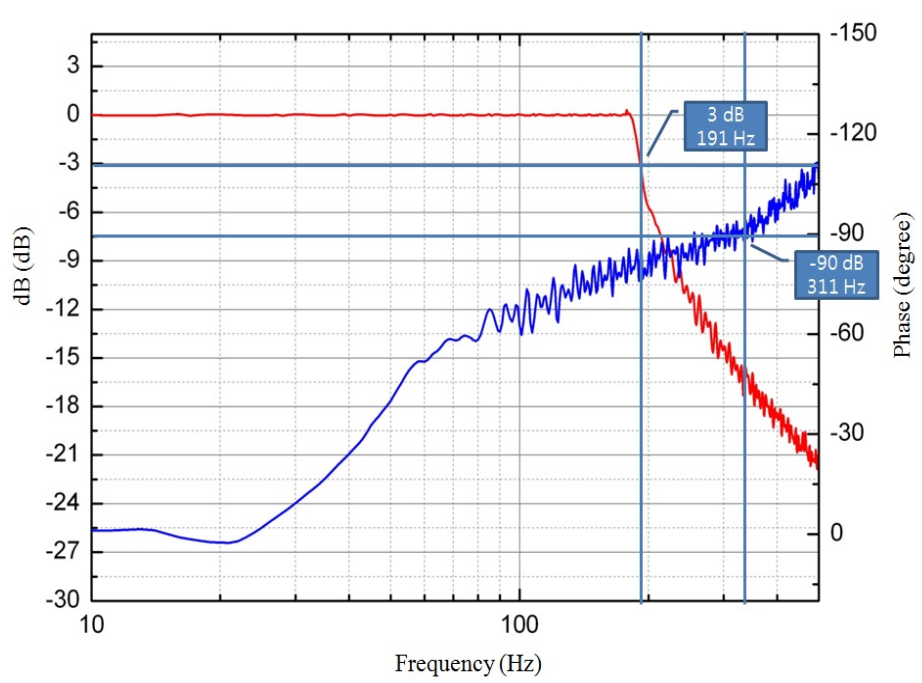

Fig. 12 Frequency response at $25 \%$ of input signal. 


\section{Conclusions}

In this paper, governing equations of solenoid actuator for valve operation and design program is composed by use of them. The main design factors derived by substitution of general design value and empirical design coefficients to the design program, and they are verified by FEM analysis. We also manufactured the prototype solenoid actuator by use of the results of the design program. And we obtained the following experimental results.

The values of deducted main design factors which are based on the theoretical governing equations are very similar to the result of FEM analysis.

Especially, the accuracy of the examined theories and design program are proved by consideration of the following: The frequency response of prototype solenoid actuator approaches the target value of $100 \mathrm{~Hz}$ as $191 \mathrm{~Hz}$ at $25 \%$ input signal. And the step response is $5 \mathrm{~ms}$. These test results mean that, the performance of the prototype solenoid actuator was satisfactory from the viewpoint of the proportional and servo type valve operation.

Hereafter, we will assemble this prototype with valve body, and will perform the general hydraulic valve tests like test of control pressure, test of control flow rate, hysteresis test and test of internal leakage. By these procedures, we will confirm the suitability of the prototype as a high speed solenoid actuator for valve operation.

\section{References}

[1] Roters, C. 1970. Electro Magnetic Device. New York: John Wiley \& Sons, Inc.

[2] Sung, B. J., Lee, E. W., and Kim, H. E. 2002. "Development of Design Program for On and Off Type Solenoid Actuator." In Proceedings of the KIEE (Korean Institute of Electrical Engineers) Summer Annual Conference 2002(B), 929-31.

[3] William H. H. 1986. Engineering Electromagnetics. New York: Mc Grawhill.

[4] Ogata, K. 1998. System Dynamics. Minnesota: Prentice Hall.

[5] Kajima, T. 1995. "Dynamic Model of the Plunger Type Solenoid at Deenergizing State." IEEE Transactions on Magnetics 31 (3): 2315-23.

[6] Nasar, S. A., and Boldea, I. 1987. Solenoid Electric Motors. New Jersey: Prentice-Hall Inc. Englewood Cliffs.

[7] Stolting, H. D., and Beisse, A. 1987. The Small Electrical Machines. Stuttgart: B. G. Teubner Stuttgart.

[8] Hydraulic and Pneumatic Lap. of KIMM (Korea Institute of Machinery \& Materials). 2001. Development of Low Consumption Power Type Solenoid Valve. KIMM-CSI (Conference Seminar of Institute) annual report

[9] Sung, B. J., Lee, E. W., and Kim, H. E. 2004. "Characteristics of Non-magnetic Ring for High-Speed Solenoid Actuator." In Proceedings of the Eleventh Biennial IEEE Conference on Electromagnetic Field Computation, 342.

[10] Kanda, K. 1997. Design Concept for DC Solenoid of Pneumatic Valve. KIMM research reporter. 